\title{
MESH-BASED ENTRY VEHICLE AND EXPLOSIVE DEBRIS RE-CONTACT PROBABILITY MODELING
}

\author{
Mark A. McPherson ${ }^{*}$ and Gavin F. Mendeck ${ }^{\dagger}$ \\ NASA Johnson Space Center, Houston, Texas, 77058
}

\begin{abstract}
The risk to a crewed vehicle arising from potential re-contact with fragments from an explosive breakup of any jettisoned spacecraft segments during entry has long sought to be quantified. However, great difficulty lies in efficiently capturing the potential locations of each fragment and their collective threat to the vehicle. The method presented in this paper addresses this problem by using a stochastic approach that discretizes simulated debris pieces into volumetric cells, and then assesses strike probabilities accordingly. Combining spatial debris density and relative velocity between the debris and the entry vehicle, the strike probability can be calculated from the integral of the debris flux inside each cell over time. Using this technique it is possible to assess the risk to an entry vehicle along an entire trajectory as it separates from the jettisoned segment. By decoupling the fragment trajectories from that of the entry vehicle, multiple potential separation maneuvers can then be evaluated rapidly to provide an assessment of the best strategy to mitigate the re-contact risk.
\end{abstract}

Subscripts

$\begin{array}{ll}D & =\text { debris fragment } \\ i & =\text { ballistic coefficient class } \\ V & =\text { entry vehicle }\end{array}$

\section{Nomenclature}

I. Introduction

$\mathrm{T}$ HE potential for re-contact of jettisoned objects with a parent spacecraft during the atmospheric entry phase represents a significant risk to both mission success and in the case of human spaceflight, the crew. This risk is greatly magnified by the potential for an explosive breakup event that may fragment the jettisoned body into a large number of smaller pieces. Each of these fragments then poses a separate risk to the vehicle and the crew. Previous

\footnotetext{
* Aerospace Engineer, Ascent/Descent Flight Dynamics Division, NASA Johnson Space Center, Member AIAA.

${ }^{\dagger}$ Aerospace Engineer, Orion Project Vehicle Integration Office, NASA Johnson Space Center, Member AIAA.
} 
re-contact risk models have sought to examine closest approaches from several Monte Carlo data sets to provide a risk assessment [1]. However, such techniques are computationally expensive, hindering iterative trajectory optimization. To better facilitate this, a probabilistic technique was developed that examines spatial debris density around a jettisoned body, as a function of time after an explosive event.

This technique takes simulated debris trajectories and discretizes the locations of each fragment into a series of volumetric elements or cells, creating a spatial debris density mesh. Using a stochastic approach, the probability of a collision between a spacecraft and any debris inside one of these cells, can then be computed, independent of the spacecraft's proximity to each individual fragment. Thus, this method provides a robust and computationally efficient means of quantifying and mitigating re-contact risk through the optimization of separation maneuvers.

\section{Simulation of Explosive Event}

In order to capture the location of fragments from the explosive event, each piece was propagated from the time of the breakup down to an altitude of $30.48 \mathrm{~km}(100,000 \mathrm{ft})$ using the Johnson Space Center's Advanced NASA Technology Architecture for Exploration Studies (ANTARES) simulation. This was modified to allow a translational force to be applied to a fragment, corresponding to a particular change in velocity associated with the explosive event. This force was then applied to each fragment in a random direction, creating a uniform spherical distribution to simulate an instantaneous explosive breakup. The resulting trajectories were then analyzed in a noninertial (moving) reference frame centered on the jettisoned body, as if it had not experienced breakup.

$$
\beta=\frac{M}{A \cdot C_{D}}
$$

The fragment properties such as mass, frontal area and drag coefficient were captured in the ballistic coefficient according to Eq. (1). Assuming a tumbling fragment, with a constant drag model and no lift or debris demise, this parameter sufficiently describes the behavior of each debris piece in the atmospheric free stream and allows for

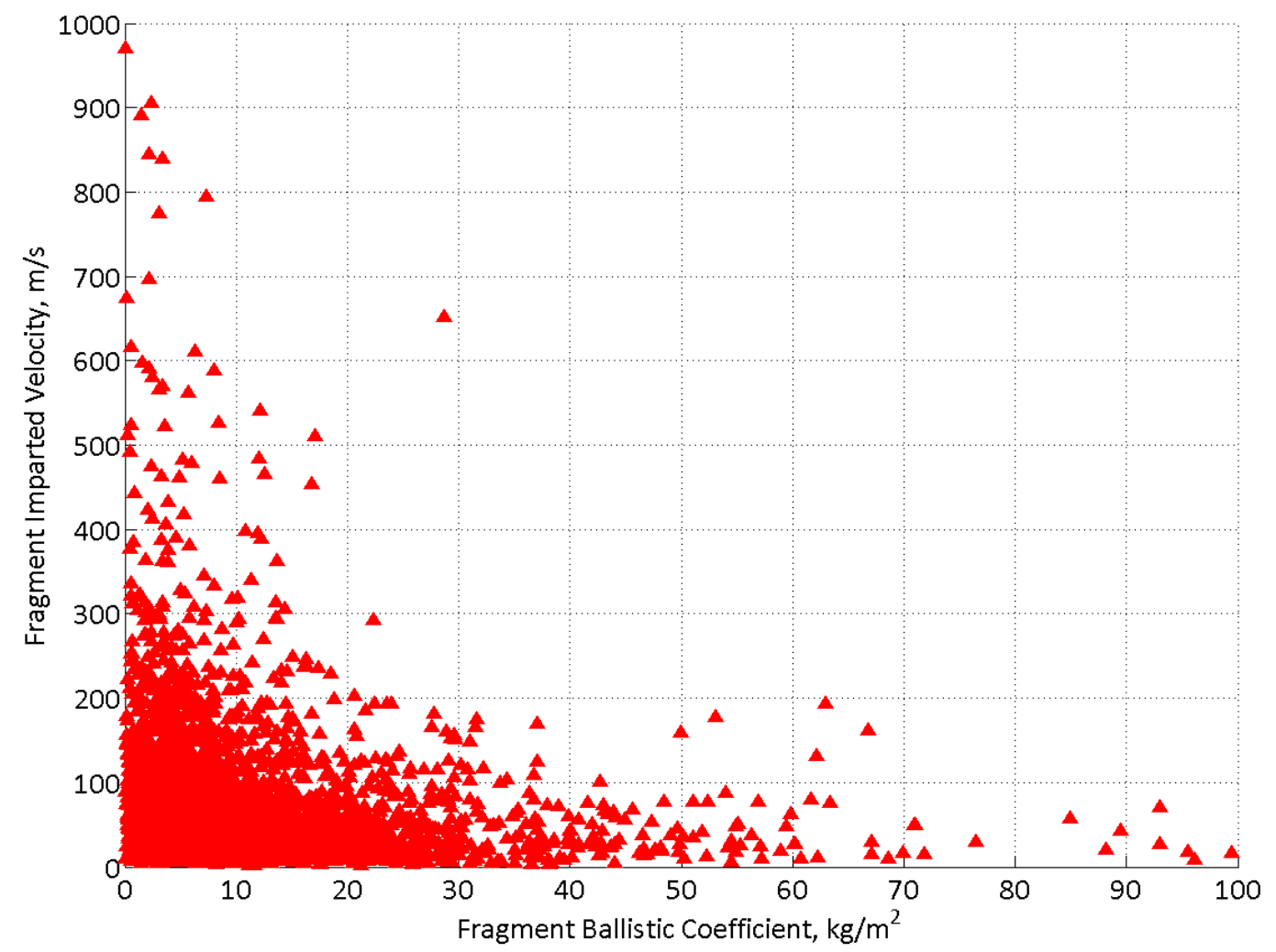

Fig. 1 NASA Standard Breakup Model imparted velocity vs. ballistic coefficient. The distribution of imparted velocities and ballistic coefficients for fragments with $L_{C}>2.24 \mathrm{~cm}, C_{D}=1$. 
propagation of its trajectory post-breakup. Here debris demise refers to the complete ablation of a fragment due to aerothermodynamic heating in the high velocity free stream.

The total number of fragments larger than a particular characteristic length, $\mathrm{L}_{\mathrm{C}}$, expected from the blast was determined from the NASA Standard Breakup Model [2], assuming an $\mathrm{L}_{\mathrm{C}}=2.24 \mathrm{~cm}$. It was also used to derive the associated distribution of both the ballistic coefficient and the magnitude of the imparted velocity for each fragment. This model incorporates empirical data from observed explosive breakups, along with ground test data to generate a probabilistic distribution of fragment area/mass ratios and imparted velocities for a given total object mass. Ballistic coefficients for each piece were then calculated as the reciprocal of the area/mass ratio, assuming for the purposes of this analysis a constant $C_{D}=1$. Since the drag coefficient will vary with altitude, as the debris move from the freemolecular to the continuum flow regimes, $\mathrm{C}_{\mathrm{D}}$ was chosen as a constant value equal to the average between the two flow regimes for a flat plate [3]. This assumes that the fragments generated by the explosive breakup can be modeled as flat plates, and that any deviations in $C_{D}$ from this average value will be captured within the dispersions of the area/mass ratio generated by the stochastic explosive breakup model. A representative distribution of ballistic coefficients and imparted velocities for all of the theoretical fragments of a generic jettisoned body after explosive breakup is illustrated in Fig. 1.

The resulting debris cloud when all of the fragments are simulated can be seen in Fig. 2. Snapshots were taken at $5 \mathrm{~s}$ and $30 \mathrm{~s}$ after the instantaneous breakup clearly showing how the pieces are distributed radially by the blast, as well as along the velocity vector by exposure to the free stream. In the moving reference frame the majority of fragments are located up-range of the origin, due to the higher ballistic coefficient of the jettisoned object prior to fragmentation. This result indicates that the highest region of debris flux is expected to occur aft of the exploding body.

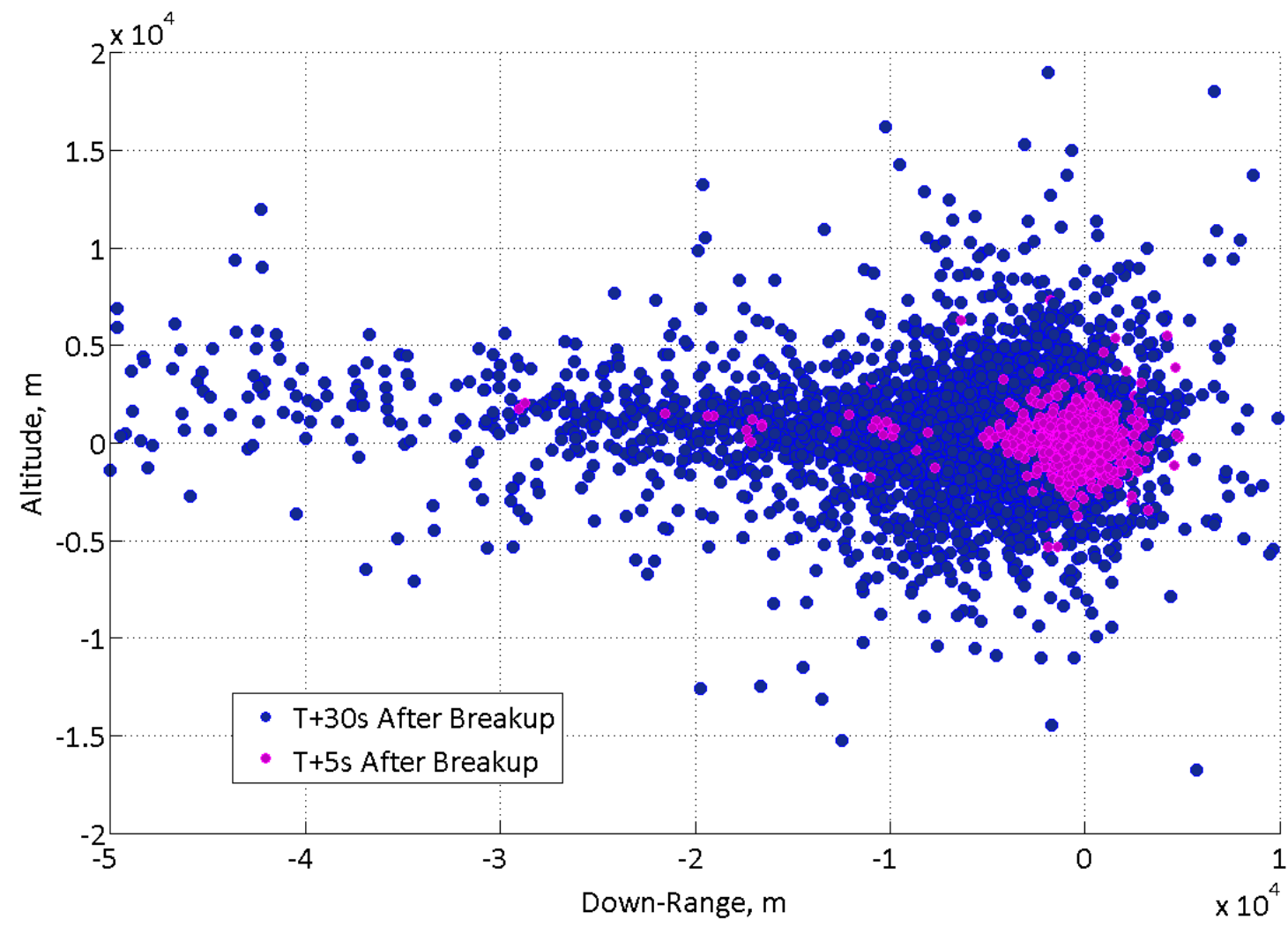

Fig. 2 Debris positions in non-inertial reference frame. The in-plane location of debris in the non-inertial reference frame at $T+5$ and $T+30$ s after breakup of a generic jettisoned body at $83.82 \mathrm{~km}(275,000 \mathrm{ft})$.

\section{Debris Distribution Maps}

While knowledge of the precise location of each fragment is useful information, for the purposes of assessing far-field re-contact risk during entry, this data is far too sparse without simulating hundreds of thousands of trajectories. In order to capture the debris cloud information without the need for massive simulations, volumetric 
elements were used. This is similar to techniques employed in molecular gas dynamics to compute the properties of rarefied flows by simulating molecular collisions inside volumetric cells [4]. However, rather than modeling collisions between molecules, here we were concerned with using these cells to predict strikes on a spacecraft. For the purposes of this work, far-field re-contact is defined as the collision of the entry vehicle with any component of the jettisoned body (intact or fragmented) after an arbitrary minimum separation distance between the two bodies has previously been achieved.

To assess this risk, the space around the entry vehicle was discretized in a non-inertial reference frame centered at the location of the exploding body, as if it had continued its trajectory intact. Each fragment was then binned into a cell according to its location at a particular point in time. The advantage of such a reference frame was that it effectively de-coupled the debris trajectories from that of the entry vehicle. As such, a generic spatial mesh, representative of the debris cloud at each point in time, for a particular breakup altitude, was able to be constructed. This could then be used to gauge the relative debris density in the region surrounding the entry vehicle and assess the resulting re-contact probability for that spacecraft, as will be illustrated in the following sections.

\section{A. Mesh Type}

The spatial distribution of debris following an explosive breakup is assumed to be initially spherical. However, depending upon the dynamic pressure experienced by the vehicle at the time of breakup the shape of the resulting debris cloud will vary with time. Therefore, for various nominal or contingency entry trajectories and breakup altitudes, different methods for discretizing the fragment locations will produce different results.

For breakup at low altitudes, the thicker atmosphere will tend to elongate the debris cloud along the vehicle's velocity vector, as the fragments with low ballistic coefficients are rapidly blown backwards in the non-inertial reference frame. This asymmetry can be captured by considering the debris cloud mesh in a cylindrical coordinate system with the longitudinal axis coinciding with the velocity vector. The volume contained inside each element will then grow as the radial distance from the longitudinal axis increases. Figure 3 illustrates such a cylindrical mesh for the representative breakup of a jettisoned body, three seconds after the explosive event. The regions of highest debris density are colored in red.

Where breakup occurs at higher altitudes in the thinner atmosphere, the effect of the free-stream on the fragments is less pronounced and therefore, the debris cloud can be described (at least initially) using a spherical coordinate system, centered at the origin of the moving reference frame. Again, the volume contained inside each elemental region will increase the farther the element is from the origin in the non-inertial frame. Figure $4 \mathrm{a}$ illustrates a spherical mesh for the same representative case modeling the explosive breakup of a jettisoned body.

A third and relatively robust mesh type that may be used in either situation is one based on a Cartesian

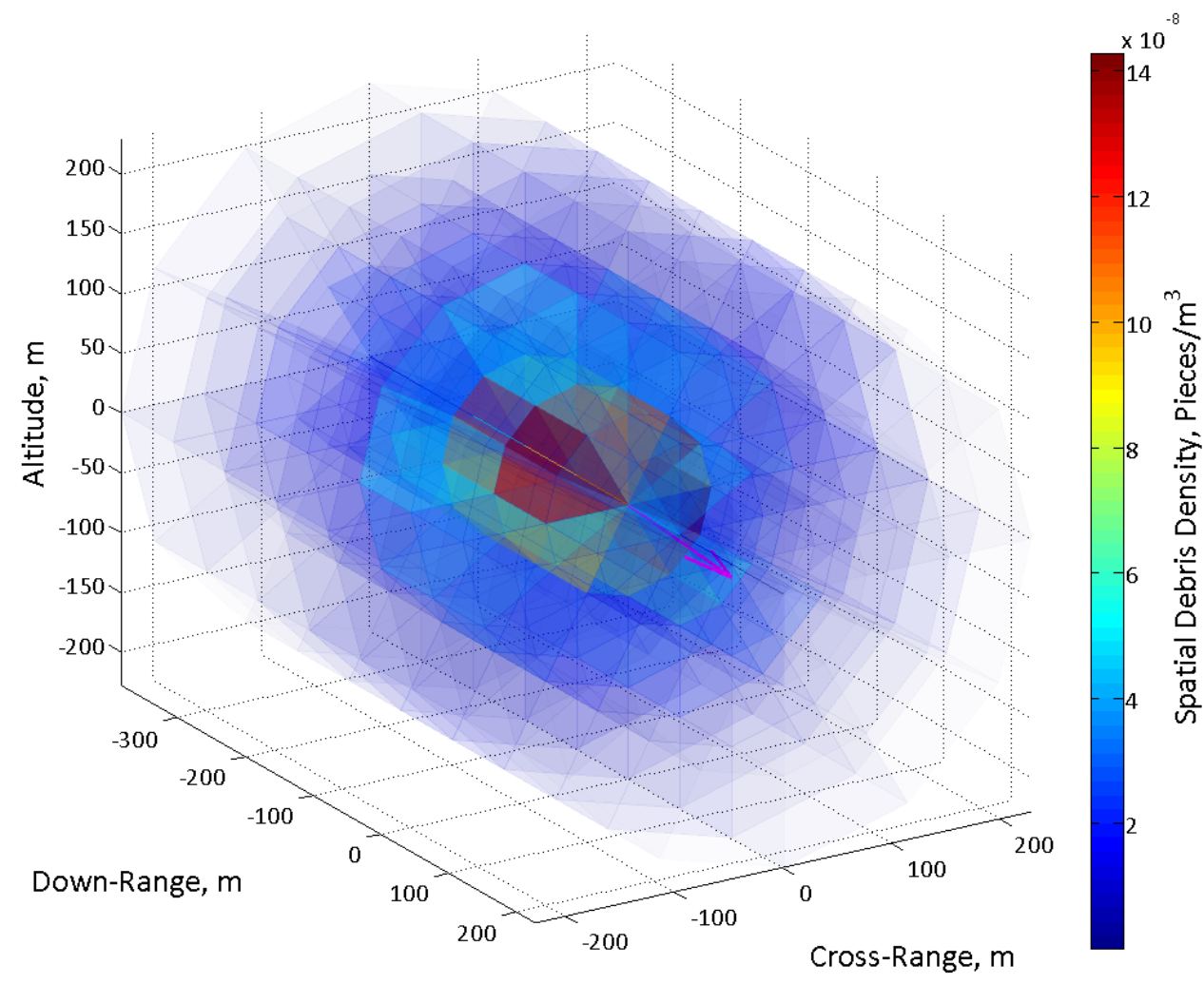

Fig. 3 Cylindrical coordinate mesh. A fixed cylindrical mesh $T+3 \mathrm{~s}$ after the simulated explosive breakup of a generic jettisoned body at an altitude of $83.82 \mathrm{~km}$ $(275,000 f t)$. The arrow indicates the direction of the velocity vector at the time of explosive breakup.

4

American Institute of Aeronautics and Astronautics 
grid with cubic volumetric elements dividing up the region around the breakup, as seen in Fig. 4b. In this case the volume of space contained inside each element is constant with the radial distance from the blast center.

Due to the evolution of the debris cloud with time, the accuracy with which each of these mesh types captures the spatial debris density will vary. In addition, depending upon the proximity of the entry vehicle to the exploding jettisoned body there may be significant differences. For close proximity, the major concern is from initial blast fragments, and as such the spherical mesh is likely to be the best choice. For greater separation distances the risk from fragments in the tail of the debris cloud may be the primary concern and as such, a cylindrical or Cartesian mesh may prove more effective.

The selection of the mesh type as well as mesh size requires some degree of experience on behalf of the analyst in order to minimize discretization errors in the model. To compare the mesh types a generic separation sequence was chosen and the length of the primary separation burn by the Reaction Control System (RCS) thrusters varied to produce a range of separation trajectories. Figure 5 illustrates how the total strike probability changes with burn time for each mesh type. As separation burn time increases, the distance between the entry vehicle and the blast center increases, generally leading to lower total re-contact probabilities. However, some discontinuities in the data are evident, particularly for the Cartesian mesh. These arise where the positions of the fragments are too coarsely captured by the mesh or insufficient fragments have been simulated, leading to large jumps in total strike probability for small changes in burn time. The steep dropoff at $9 \mathrm{~s}$ for the Cartesian mesh in Fig. 5 is due to the cells containing the entry vehicle being insufficiently large, at that distance from the blast, to encompass enough fragments to accurately represent the debris density in that region. For the cylindrical and spherical meshes the volume of the cells grows with distance from the blast center and therefore, they do not exhibit the same degree of variability.

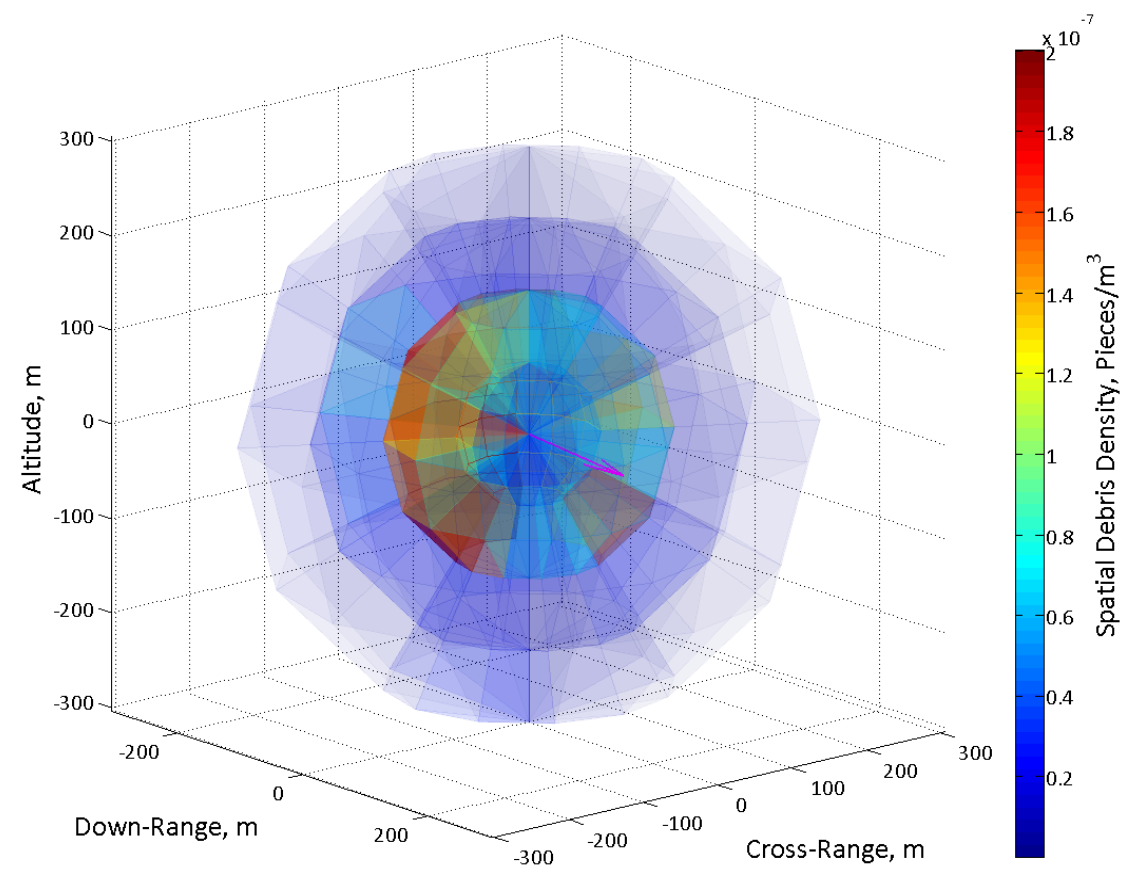

a)

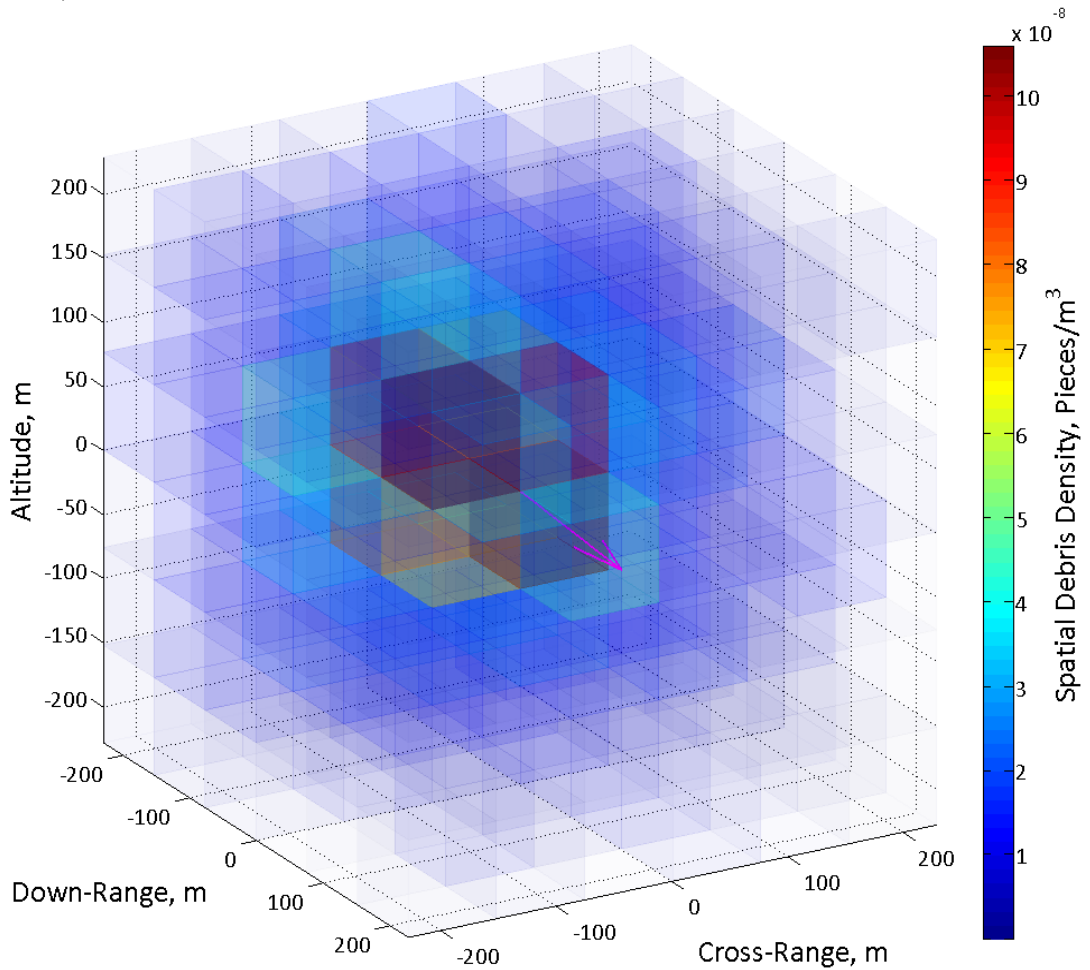

b)

Fig. 4 Spherical \& Cartesian coordinate meshes. Fixed spherical (a) and Cartesian (b) meshes at $T+3 \mathrm{~s}$ after the simulated explosive breakup of a generic jettisoned body at an altitude of $83.82 \mathrm{~km}(275,000 \mathrm{ft})$. 


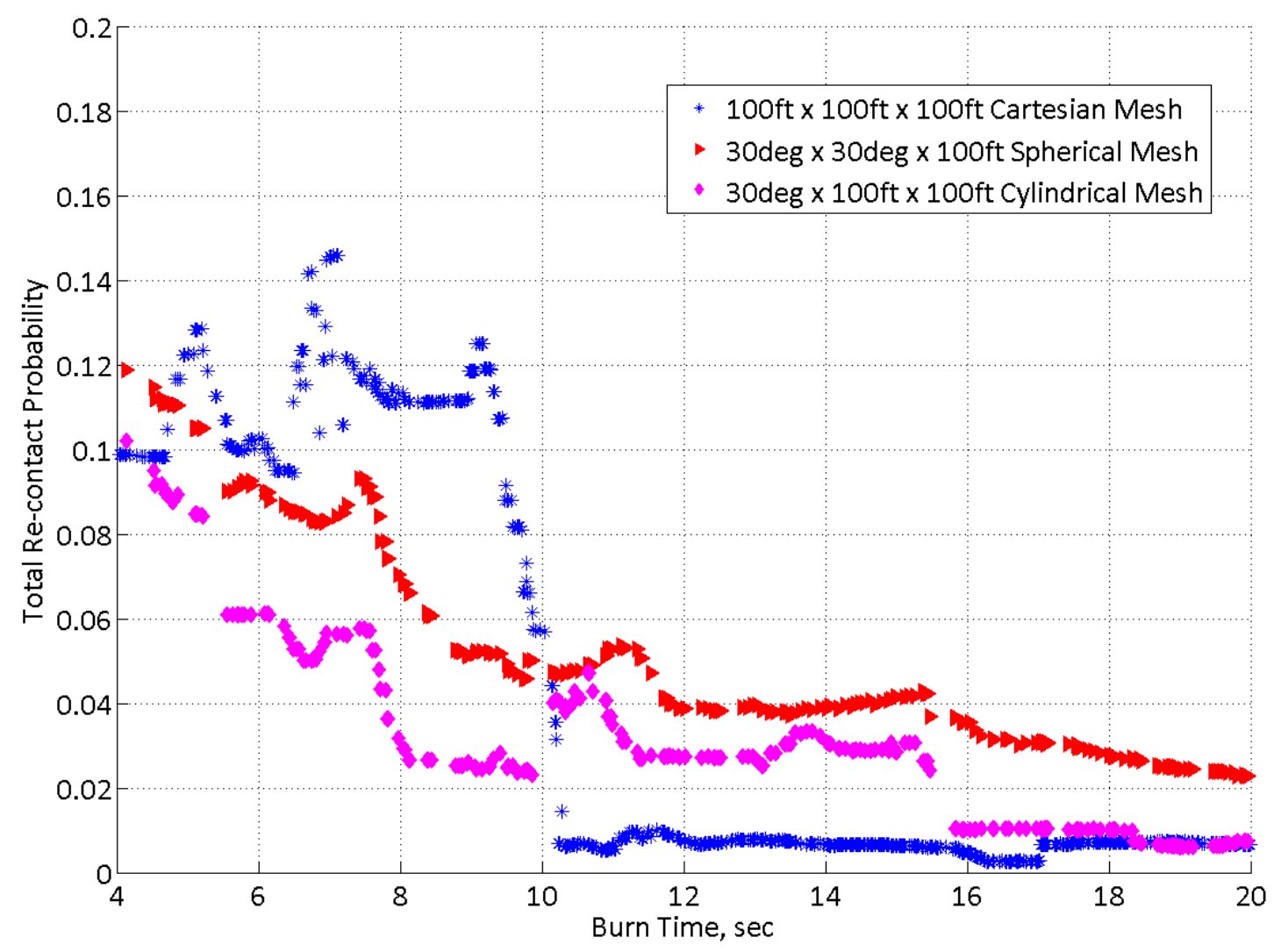

Fig. 5 Total strike probabilities for different mesh types. Illustrates the trend in strike probability for the three different mesh types as the length of a separation burn is varied for a generic separation sequence where explosive breakup occurs at an altitude of $83.82 \mathrm{~km}(275,000 \mathrm{ft})$.

\section{B. Mesh Size}

Selection of the dimensions of the mesh elements is an important aspect of the analysis because, as with mesh type, discretization errors tend to be amplified by inappropriate choices. The appropriate dimensions are dependent on the particular breakup case considered. However, in general, sufficient accuracy can be achieved by ensuring that the cells are large enough such that the majority of fragments do not travel more than one cell in a single time step, and each cell contains at least a few particles on average [5]. Again the proximity of the entry vehicle to the blast center at the time of breakup is an important factor to consider. The closer the entry, the finer the mesh needed to accurately capture the shape of the debris cloud as it encompasses the spacecraft. Two methods of setting the mesh size were considered in this paper: a 'fixed' and an 'adaptive' method.

A 'fixed' dimension mesh is relatively simple to implement and specifies a fixed spatial dimension for each of the coordinates. The mesh region is then defined as a fixed region of Euclidean space encompassing sufficient debris fragments for assessment of re-contact with the entry vehicle. Limitations can then easily be placed upon the cells such that fragments do not move more than one cell's width in a time step. However, due to the natural growth of the debris cloud over time, these dimensions can be difficult to select and the debris mesh itself can become large and unwieldy. In addition, as the debris move outwards, if insufficient pieces were simulated, some cells may become sparsely populated with fragments leading to discretization errors as seen in Fig. 5. This can be overcome by simulating more debris fragments. However, it requires significantly more computational time, as the number of fragments necessary to fill a fixed volume cell increases proportionally with the cube of its distance from the blast center.

Using an 'adaptive' dimension mesh that grows with the debris cloud allows many of these issues to be avoided. This was achieved by setting the maximum dimensions of the mesh, in both the radial and longitudinal directions, to be equal to the distance that the 99th percentile fragment has travelled along that coordinate at a particular instant in time. As the debris move away from the blast center the volume contained inside each mesh element will then grow to encompass more fragments. This tends to reduce many of the discretization effects seen with a fixed size mesh. A comparison between a fixed and an adaptive cylindrical debris mesh for the parametric sweep of burn times can be seen in Fig. 6. 


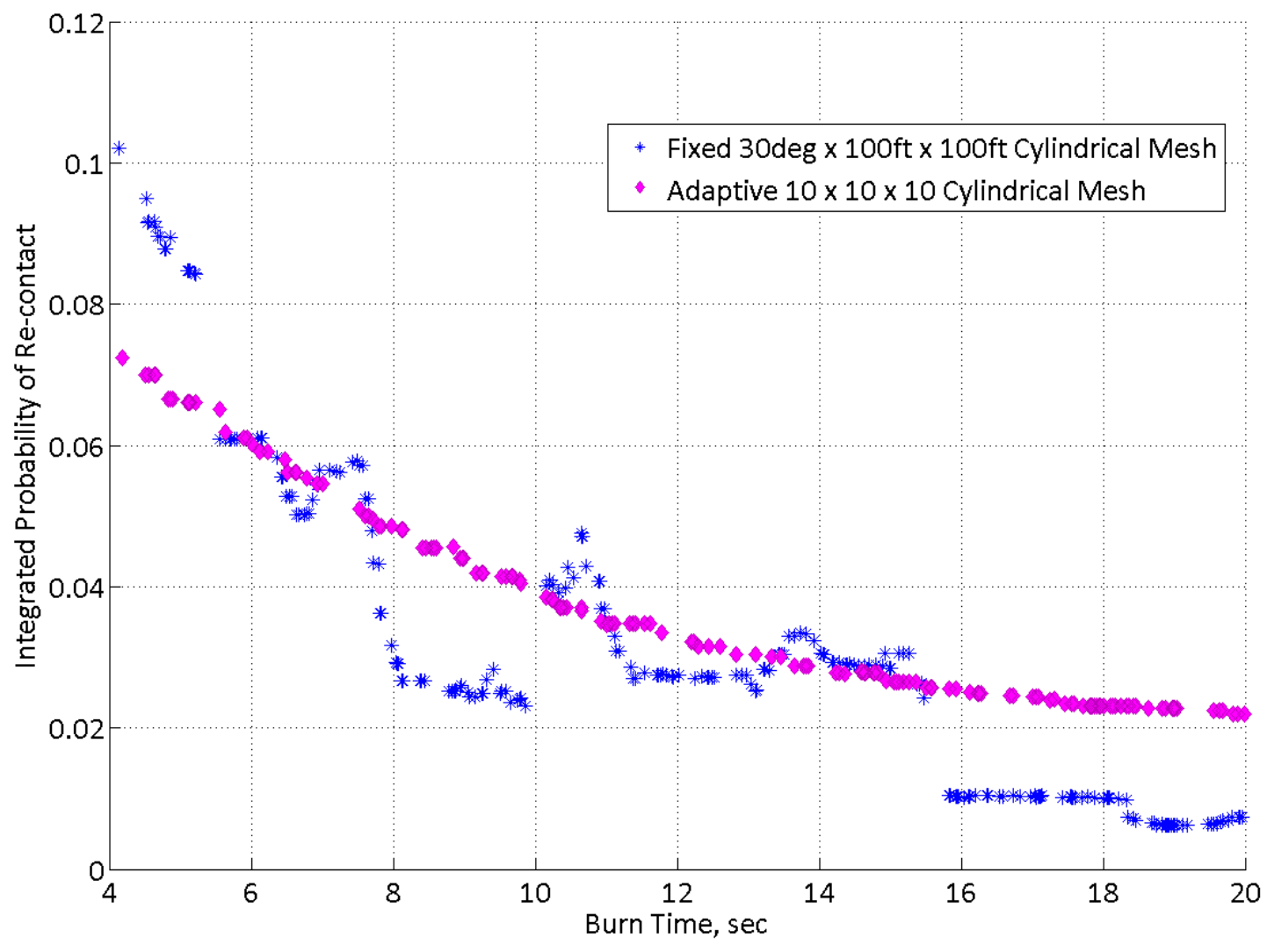

Fig. 6 Strike probabilities for different mesh types. Illustrates the trend in strike probability for a fixed vs. adaptive cylindrical mesh as the length of a separation burn is varied for a generic separation trajectory with breakup occurring at an altitude of $83.82 \mathrm{~km}(275,000 \mathrm{ft})$.

\section{Debris Weighting}

Due to the stochastic nature of an explosive breakup event, it was necessary to model many more fragments than would be expected to be produced. This was done to effectively simulate the entire region of space that could potentially be occupied by debris from such a breakup. In order to correct for this difference in the number of simulated versus actual debris fragments, the spatial debris density inside each volumetric cell was weighted according to Eq. (3). The parameter $\eta$ is a weighting factor based on the classification of each fragment into one of ten equal intervals of ballistic coefficients called the 'ballistic coefficient class.' This weighting factor is calculated according to Eq. (4) as the ratio of the actual number of fragments of that class, divided by the simulated number of that class. This is based on the simulation of a uniform distribution of fragment ballistic coefficients between the minimum and maximum values, and assumes all fragments are ejected in random directions by the blast. For example, in the case of high ballistic coefficient pieces, of which fewer are expected to be produced, the weighting factor would be very low. Each fragment would then represent a fraction of a whole piece when calculating debris density inside a particular cell. This reduces statistical scatter and ensures that the debris cloud is more representative of all the possible locations of the debris pieces ejected by the explosive event.

$$
\begin{gathered}
\bar{\rho}_{D}=\sum_{i=1}^{n} \eta_{i} \cdot \rho_{D, i} \\
\eta_{i}=\frac{N_{A, i}}{N_{S, i}}
\end{gathered}
$$

An additional benefit of weighting debris fragments is that debris distributions can be tailored according to the type of breakup event without the need to re-simulate the trajectories of each piece. For example, when considering 


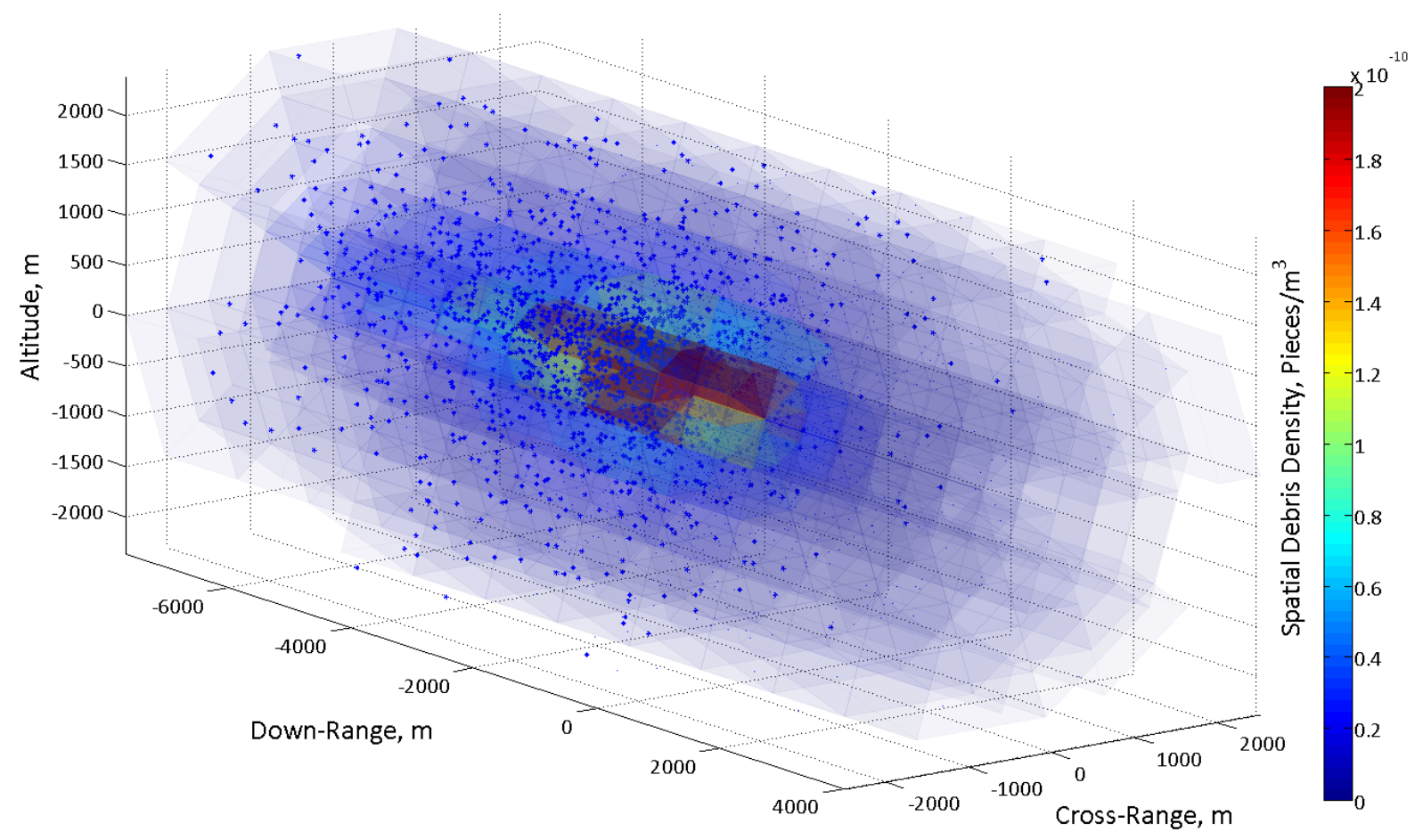

Fig. 7 Adaptive cylindrical mesh with debris fragments. The location of simulated debris fragments from a generic jettisoned object overlaid onto an adaptive cylindrical debris density mesh $15 \mathrm{~s}$ after breakup.

high-energy versus low-energy explosive events, the distribution of the fragment ballistic coefficients will vary. By simply manipulating the debris weighting factor, both of these cases, as well as every case in between, could then be represented using the same set of fragment trajectories.

\section{Stochastic Approach to Re-contact}

A stochastic model for assessing re-contact risk assumes that the probability of a particular fragment from an explosive event coming into contact with the entry vehicle is low and as such, it is a rare event. In addition, for the purposes of this model, any strike on the entry vehicle by debris is considered a failure. Combined with the assumption that the probability of such a collision occurring in one time interval is independent of it occurring in another, this scenario can be modeled as a Non-Homogeneous Poisson Process (NHPP). The advantage of this statistical approach is that it reduces the sensitivity of the answer to the number of cases run. In Monte Carlo analysis, a large number of runs are necessary to sufficiently capture a rare event such as a debris strike. The accuracy of the computed probabilities is then proportional to the number of runs. Using a NHPP to model these events reduces this dependency, allowing the re-contact probabilities to be potentially assessed with far fewer runs.

\section{A. Debris Flux}

From methods in molecular gas dynamics the collision probability between two particles over a finite time interval can be calculated as "the ratio of the volume swept out by their total cross-section, moving at the relative speed between them, to the volume of the cell" [4]. More simply this can be thought of as the debris flux $\varphi_{D}(t)$ the vehicle is exposed to at each instant following the explosive event [6]. Using the spatial mesh, this can be calculated inside each cell as the product of the scaled debris density and the magnitude of the average relative velocity between the debris and the vehicle according to Eq. (4). The relative velocity vector, from Eq. (5), is the velocity vector of the debris in the inertial frame minus the velocity vector of the entry vehicle [7], as shown in Fig. 8.

$$
\begin{gathered}
\varphi_{D}(t)=\bar{\rho}_{D}(t) \cdot\left|\vec{V}_{R}(t)\right| \\
\vec{V}_{R}(t)=\vec{V}_{D}-\vec{V}_{V}
\end{gathered}
$$


From Eq. (3). the weighted spatial density represents the weighted number of fragments that occupy a particular cell in the mesh at any point in time, divided by the total volume of that cell. This implicitly assumes that the fragments inside each cell are randomly distributed such that the spatial density is uniform throughout. This is a reasonable assumption given an appropriate mesh type and resolution are selected, and that sufficient fragments have been simulated. Figure 9 plots both the spatial debris density and relative velocity seen by the entry vehicle along a generic separation trajectory. In this example the debris density experienced by the vehicle is greatest shortly after the blast at 202s elapsed time, and decreases as the debris cloud expands outwards. Debris-vehicle average relative velocity continues to rise following the explosive event as the lower ballistic coefficient pieces are blown back toward the entry vehicle. The offset time between the explosive event and the entry vehicle's first encounter with debris is due to the initial separation distance of approximately $200 \mathrm{~m}(656 \mathrm{ft})$ between the vehicle and the jettisoned body at the time of the blast.

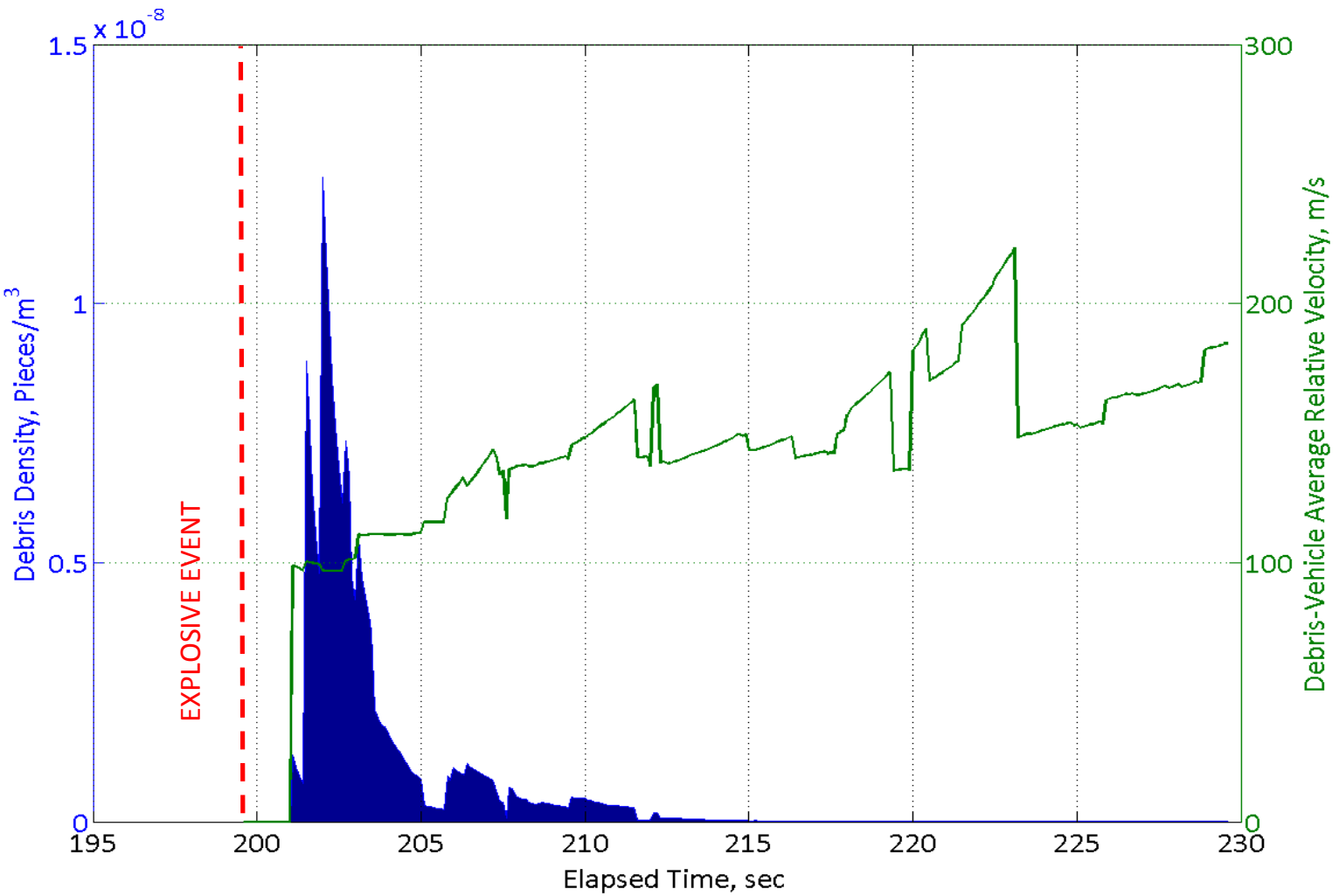

Fig. 9 Spatial debris density and relative velocity. Spatial debris density and relative velocity between the debris and entry vehicle as a function of time along a generic trajectory.

9

American Institute of Aeronautics and Astronautics 


\section{B. Integrated Collision Probability}

Modeling the entry vehicle as a sphere with a uniform collision cross-section removes the dependency of the results on the direction of the debris flux. The intensity parameter in the Poisson process can then be calculated using Eq. (6) from the product of the non-directional flux term and the area of the entry vehicle [6]. From [8], this can then be integrated as a function of time using Eq. (7) over the interval $\left[\mathrm{t}_{1}, \mathrm{t}_{2}\right]$ to give the cumulative intensity parameter for re-contact along a particular trajectory.

$$
\begin{gathered}
\lambda(t)=\varphi_{D}(t) \cdot A_{V} \\
\Lambda=\int_{t_{1}}^{t_{2}} \lambda(\tau) d \tau
\end{gathered}
$$

In a Poisson process the probability of $\mathrm{k}$ events occurring in a given interval is determined by Eq. (8). Subtracting this from one, where $\mathrm{k}$ is zero, the probability of one or more debris strikes along a trajectory can then be calculated using the cumulative intensity parameter, Eq. (9).

$$
\begin{aligned}
& \operatorname{Pr}\{X=k\}=\frac{(\Lambda)^{k} e^{-\Lambda}}{k !} \\
& \Rightarrow \operatorname{Pr}\{X \geq 1\}=1-e^{-\Lambda}
\end{aligned}
$$

Thus, for a given vehicle $A_{V}$, the probability of at least one debris strike occurring at some point along a particular separation trajectory will depend solely upon the cumulative debris flux the vehicle experiences along that trajectory. Figure 10 illustrates how the re-contact probability accumulates over time for a set of four different

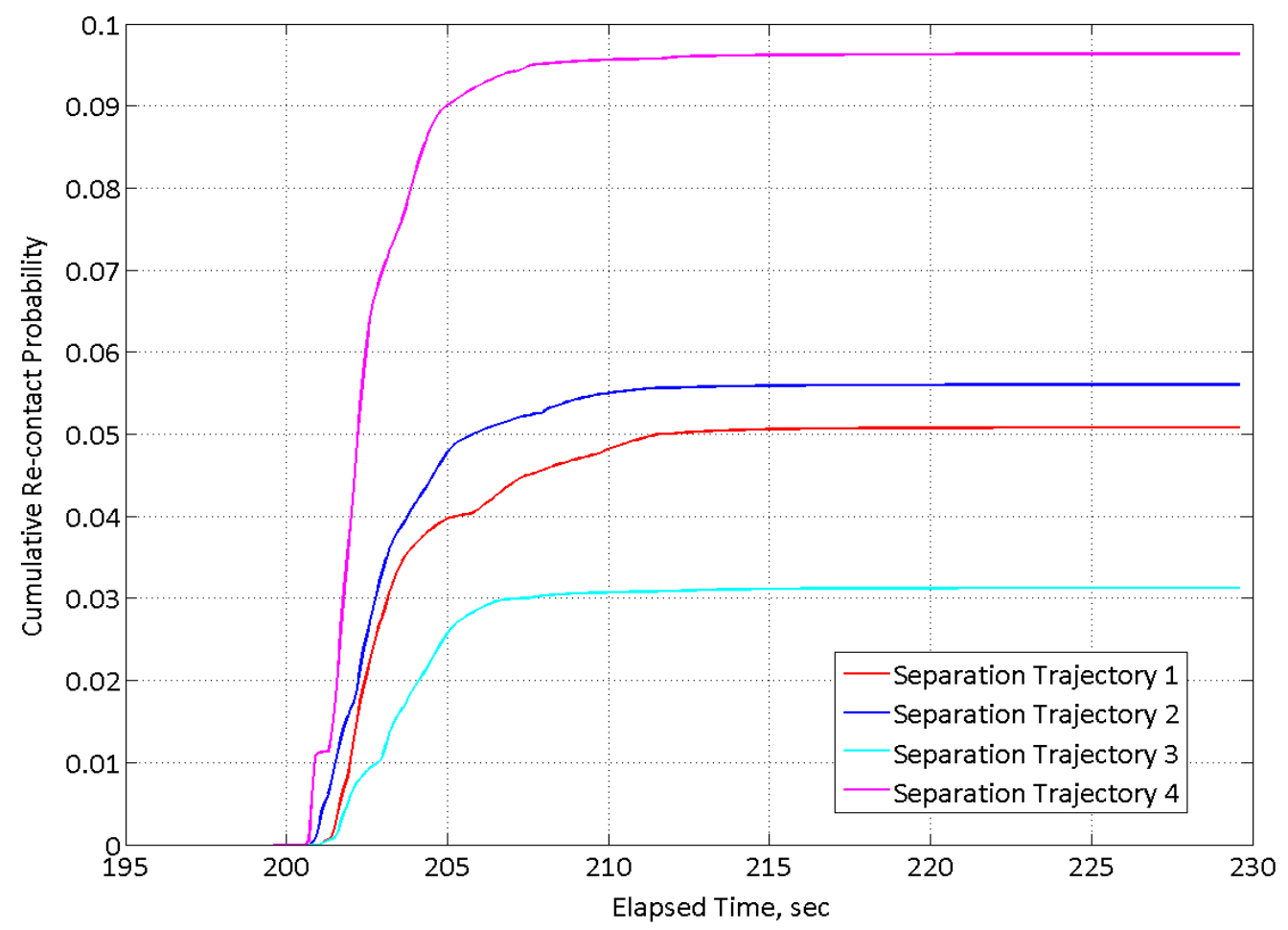

Fig. 10 Cumulative re-contact probability along various trajectories. Cumulative re-contact probabilities as a function of time along four different generic separation trajectories.

10

American Institute of Aeronautics and Astronautics 
separation trajectories. This figure shows how, for this example, the majority of the risk to the entry vehicle occurs within the first five seconds following the explosive breakup. Note these are generic separation trajectories and are not optimized for minimum re-contact probability.

\section{Separation Trajectory Design}

As illustrated, a stochastic approach to re-contact allows a total probability of collision to be assigned to a particular separation trajectory. However, the major advantage of the approach presented in this paper over other methods lies in the decoupling of the debris cloud from these trajectories. By referencing the entry vehicle's position in a non-inertial frame, assessment of the re-contact probability associated with each separation trajectory can be performed more rapidly using the debris mesh. This implicitly assumes that small variations in the altitude at breakup will not have a large impact upon the distribution of the debris fragments, relative to the moving reference frame. A parametric sweep of jettison attitudes and burn times can then be performed to reveal particular separation maneuvers that best mitigate the re-contact risk to the entry vehicle. This can be done while including atmospheric, gravitational, navigation, and control system dispersions in order to capture any uncertainty associated with the separation maneuver. Utilizing this technique, hundreds of possible separation maneuvers may be analyzed with a single debris mesh, allowing the separation trajectory to be rapidly optimized. It must be noted that while small variations to breakup altitude do not necessitate a new debris mesh; simulating large variations, such as those inherent when predicting a random event like an explosive breakup, will require a new mesh. Nevertheless, this technique has the potential to greatly simplify post-separation maneuver design.

\section{Conclusions and Forward Work}

The capability to rapidly quantify the debris re-contact probability for a spacecraft arising from the explosive breakup of jettisoned components is valuable for ensuring mission success and crew safety. Utilizing this spatial discretization technique allows the regions of high and low relative probability for an entry vehicle to be clearly visualized. In addition, such a stochastic method provides the means to quantify an absolute risk to the vehicle, integrated over its entire trajectory, so as to satisfy operational safety requirements. Combined, these elements enable safer design and operation of crewed and un-crewed spacecraft. However, this technique is highly dependent on the selection of the mesh used to spatially divide the region as well as the size of the individual mesh elements. As such, considerable care must be given to defining these parameters in order to ensure that discretization errors do not distort the results.

Future work is necessary to quantify the uncertainty associated with modeling the re-contact problem as a stochastic process in order to appropriately bound the estimates of total strike probability. Refinement of the conservative assumption that any debris strike is a failure, by inclusion of a consequence of re-contact metric, would also allow the true risk to the vehicle to be computed. Additionally, the incorporation of a fragment demise model would be particularly beneficial for assessing higher velocity entry scenarios, such as return from beyond Low Earth Orbit (LEO) missions.

\section{References}

[1] Cutri-Kohart, R. M., Graybeal, S. R., Mendeck, G. F., and Mrozinski, R. B., "Entry Vehicle and Jettisoned Debris Recontact Analysis," AIAA Atmospheric Flight Mechanics Conference and Exhibit, AIAA Paper 2003-5392, Aug. 2003.

[2] Johnson, N. L., Krisko, P. H., Liou, J.-C., and Anz-Meador, P. D., "NASA's New Breakup Model of Evolve 4.0" Advances in Space Research, Vol. 28, No. 9, 2001, pp. 1377-1384.

[3] Smith, R. N., Dobarco-Otero, J., Bledsoe, K. J., and De Laune, R. M., "User's Guide for Object Reentry Survival Analysis Tool (ORSAT) - Version 6.0", JSC-62861, 2006.

[4] Bird, G. A., Molecular Gas Dynamics and the Direct Simulation of Gas Flows, Clarendon Press, Oxford, England, 1994, Chaps. 1, 9-11.

[5] Maltsev, R. V., "On the Selection of the Number of Model Particles in DSMC Computations," 27th International Symposium on Rarefied Gas Dynamics 2010, American Institute of Physics, Melville, NY (submitted for publication)

[6] Karman Sciences Corporation, "On-Orbit Collision Hazard Analysis in Low Earth Orbit Using the Poisson Probability Distribution,” FAA Office of Commercial Space Transportation Licensing and Safety Division, Aug. 1992.

[7] Matney, M., "Dynamic Orbital Debris Collision Risk Mitigation," 55th International Astronautical Congress 2004, Vancouver, Canada, IAC-04-IAA.5.12.5.11, 2004.

[8] Leemis, L. M., "Nonparametric Estimation of the Cumulative Intensity Function for a Nonhomogeneous Poisson Process," Management Science, Vol. 37, No. 7, July 1991, pp. 886-900.

[9] Wang, L.-Q., and Stark, J. P. W, "DSMC Space Debris Simulation and Comparison with LDEF Impact Experiments," Proceedings of the Second European Conference on Space Debris, SP-393, ESA, May 1997. 
[10] Hou, S., Are, S., and Schmidt, D. P., "A Generalized Adaptive Collision Mesh for Multiple Injector Orifices," International Multidimensional Engine Modeling User's Group Meeting, Detroit, 2003.

[11] Barrows, S. P., and Swinerd, G. G., "Review of Debris-Cloud Modeling Techniques," Journal of Spacecraft and Rockets, Vol. 33, No. 4, Jul. 1996, pp. 550-555.

[12] Koppenwallner, G., Fritsche, B., Lips, T., Martin, T., Francillout, L., and De Pasquale, E., "Analysis of ATV Destructive Re-Entry Including Explosive Events," Proceedings of the Fourth European Conference on Space Debris, SP-587, ESA, Aug. 2005 . 